\title{
OBSERVATIONAL SIGNATURES OF CONVECTIVELY DRIVEN WAVES IN MASSIVE STARS
}

\author{
C. Aerts ${ }^{1,2}$ AND T. M. Rogers 3,4 \\ ${ }^{1}$ Instituut voor Sterrenkunde, KU Leuven, Celestijnenlaan 200D, 3001 Leuven, Belgium
${ }^{2}$ Department of Astrophysics/IMAPP, Radboud University Nijmegen, 6500 GL Nijmegen, The Netherlands \\ ${ }^{3}$ Department of Mathematics and Statistics, Newcastle University, Newcastle upon Tyne, UK \\ ${ }^{4}$ Planetary Science Institute, Tucson, AZ 85721, USA \\ Received 2015 April 30; accepted 2015 May 23; published 2015 June 19
}

\begin{abstract}
We demonstrate observational evidence for the occurrence of convectively driven internal gravity waves (IGWs) in young massive O-type stars observed with high-precision CoRoT space photometry. This evidence results from a comparison between velocity spectra based on two-dimensional hydrodynamical simulations of IGWs in a differentially rotating massive star and the observed spectra. We also show that the velocity spectra caused by IGWs may lead to detectable line-profile variability and explain the occurrence of macroturbulence in the observed line profiles of OB stars. Our findings provide predictions that can readily be tested by including a sample of bright, slowly and rapidly rotating OB-type stars in the scientific program of the K2 mission accompanied by highprecision spectroscopy and their confrontation with multi-dimensional hydrodynamic simulations of IGWs for various masses and ages.
\end{abstract}

Key words: asteroseismology - line: profiles - stars: massive - stars: oscillations - techniques: photometric waves

\section{INTRODUCTION}

The existence of high-order gravity-mode (g-mode) oscillations in slowly pulsating B (SPB) stars, which are corehydrogen-burning stars with masses roughly between 3 and 7 $M_{\odot}$ (hereafter SPBs; Waelkens 1991), was established more than two decades ago, prior to understanding their excitation mechanism. We now know that these oscillations are driven by the heat mechanism associated with the opacity bump due to iron-group elements in the stellar envelope (Dziembowski et al. 1993; Gautschy \& Saio 1993). However, the detection of g-mode period spacings associated with those standing waves, as predicted from theory (Tassoul 1980) and required for asteroseismology of such stars, remained impossible from the ground, even after carefully planned long-term dedicated campaigns (Aerts et al. 1999; De Cat \& Aerts 2002). This is partly due to the low amplitudes of g-modes and is also made difficult because the periods of g-modes in massive stars are of the order of days and these timescales have strong contamination by daily aliases in the amplitude spectra of ground-based photometry and high-resolution spectroscopy. g-mode asteroseismology of SPBs only saw its birth due to the uninterrupted high-precision space photometry assembled with the CoRoT and Kepler missions. These data led to the detection of period spacings caused by dipole modes of consecutive radial order and offered the mode identification necessary for seismic modeling (Degroote et al. 2010; Pápics et al. 2012, 2014, 2015).

While heat-driven g-mode oscillations in SPBs are strictly periodic and have a well-known and quantifiable effect on observed time-series photometry and spectral line-profile variations (LPVs; e.g., De Cat \& Aerts 2002; Aerts et al. 2014), the effect of waves excited stochastically by core convection (Belkacem et al. 2010; Samadi et al. 2010; Shiode et al. 2013) on such observations is relatively unknown. This lack of observational diagnostics connected with internal gravity waves (IGWs) is particularly relevant in the context of the variability of O-type stars, in which heat-driven modes are not excited, while their large convective cores likely drive IGWs efficiently. This work is therefore focused on the search for IGWs driven by core convection in photometric and spectroscopic observations of a few carefully selected observed O-type stars. Such signatures are important because the existence of these waves could point to enhanced angular momentum transport and chemical mixing, and hence guide inclusion of these processes into future theoretical models.

\section{MODELING CONVECTIVELY DRIVEN WAVES}

There have been relatively few theoretical predictions for the spectra and amplitudes of IGWs excited by core convection in massive stars. On the one hand, Browning et al. (2004) provided the first 3D simulations of the core convection for a $2 M_{\odot}$ star, but these only covered the inner $30 \%$ in radius, and hence omitted much of the wave propagation region. More recent theoretical models do consider the entire radius of the star but are limited to one-dimension (1D), neglect rotation, and must make assumptions about the nature of convection and convective-overshoot (Samadi et al. 2010; Shiode et al. 2013). Generally, those theoretical models assume Reynolds stresses in the convection zone that generate waves with a predominant frequency given by the convective turnover frequency and with frequency and wavelength spectra dictated by the assumed properties of the turbulence. The amplitudes of the waves are determined by the efficiency with which energy is transferred from convection to waves and are highly dependent on assumptions about the convective-radiative interface. Despite these shortcomings, the theoretical spectra of IGWs predicted by Samadi et al. (2010) and Shiode et al. (2013) are consistent with the observed frequency ranges of variable $\mathrm{OB}$ stars. However, their amplitudes vary significantly and appear to be inconsistent with observations. For example, the amplitudes predicted in Shiode et al. (2013) are more than an order of magnitude lower than those predicted in Samadi et al. (2010), and even those are too small to explain the detection of stochastically excited gravito-inertial modes in $\mathrm{HD}$ 
51452 by Neiner et al. (2012). These theoretical models also assume that the star is non-rotating and it was shown by Mathis et al. (2014) that the Coriolis force has a severe effect on the stochastic excitation of waves, and hence their surface amplitudes. In reality, the waves in stars are mixed gravito-inertial waves, and this should be accounted for in theoretical predictions of surface wave amplitudes. ${ }^{5}$ Beyond their effect on photometric and spectroscopic observations, these mixed type waves likely lead to substantial angular momentum transport (Rogers et al. 2013) and may be responsible for the Be star phenomenon (Ando 1986; Rogers et al. 2013; Lee et al. 2014), which could not be fully captured in earlier 1D analytic models. Clearly, observational constraints on the detection of IGWs, as well as more realistic multi-D predictions of their observational signature, would be highly beneficial.

Here, in order to make comparisons between theoretical predictions of IGWs in massive stars and observations, we use the velocity spectra from the two-dimensional (2D) numerical simulations of convectively driven waves in a $3 M_{\odot}$ star, as described in Rogers et al. (2013). While having many shortcomings (dimensionality, degree of turbulence), these simulations provide a more realistic benchmark for comparison because they self-consistently generate the waves by convection, including all nonlinearity and rotation. In order to carry out such simulations, some sacrifices had to be made. Perhaps the most relevant for the current work are that the simulations used an enhanced thermal diffusivity for numerical stability and considered only one mass. In order to compensate for the enhanced thermal damping of the waves on their journey to the stellar surface, the waves were driven harder by using an (equivalently) enhanced heat flux. While this is not a perfect representation of the waves, it is likely the best that can be done with current computational resources. Because of this shortcoming, wave amplitudes produced by these simulations should be treated with some caution. Furthermore, the simulations only considered one mass, and it is well known that the IGW frequencies depend weakly on the mass of the star (e.g., Aerts et al. 2010; Shiode et al. 2013). Repeating the simulations for various masses and evolutionary stages is beyond the scope of the current work, given that each 2D simulation takes approximately 100,000 processor hours for one set of parameters (mass, age, rotation, diffusion coefficients, luminosity). Such a simulation study will be the subject of future research. In order to compensate for the enhanced thermal diffusion, the convective heat flux adopted in Rogers et al. (2013) was typically $10^{4}$ times higher than that of a $3 M_{\odot}$ star, so the predicted spectra of IGWs might appropriately resemble those of $\sim 30 M_{\odot}$ stars since the wave spectrum is predominantly determined by properties of the convection, but this has yet to be investigated. In order to account for variations in mass with respect to the $3 M_{\odot}$ and for the too high convective flux adopted for the currently available numerical simulations, we apply one multiplicative factor (an unknown parameter) to scale the amplitudes of the waves and another one to rescale frequencies for comparisons with real stars, as discussed in more detail below. Despite these caveats, we expect that the overall characteristics and shape of the predicted frequency spectra should offer a realistic representation of the effect of IGWs on observables.

\footnotetext{
5 For ease of presentation in the following comparisons, we will use IGWs generically to mean convectively driven mixed gravito-inertial waves, sometimes referred to as GIWs.
}

In Figure 1, we show velocity spectra for three cases from Rogers et al. (2013): a non-rotating star, a rigidly rotating star with an initial rotational frequency of $1.100 \mathrm{day}^{-1}$, and a differentially rotating star whose core rotates initially with a factor 1.5 times faster than its envelope, the surface rotational frequency being $0.275 \mathrm{day}^{-1}$. These cases correspond with the simulations labeled as U1, U8, and D11 in Table 1 of Rogers et al. (2013), to which we refer for details. Using a radius from the prototypical SPB KIC 10526294 with $M=3.25 M_{\odot}$ and $R=2.215 R_{\odot}$ (Moravveji et al. 2015), the two rotating models give initial equatorial rotation velocities of 31 (non-rigid, D11) and $123 \mathrm{~km} \mathrm{~s}^{-1}$ (rigid, U8). The velocity spectra were obtained after more than 100 rotational cycles (for U8 and D11), in a layer near the stellar surface at $99 \%$ in radius, and the tangential velocity is an average over $\varphi \in[0,2 \pi]$. In Figure 1, it can be seen that the vertical velocity is many orders of magnitude smaller than the tangential velocity, as is expected for gravity waves. Figure 1 also clearly illustrates that non-rigid rotation (D11) inside the star shifts the power to lower frequencies compared to the rigidly or non-rotating cases. Given that the few estimates of core-to-envelope rotation of massive stars point to non-rigid rotation with a factor of two to five faster core than envelope (e.g., Aerts et al. 2003; Pamyatnykh et al. 2004), we use D11 for comparisons with observations.

Beyond the uncertainties in wave amplitudes and frequencies discussed above, it is not entirely clear how to convert velocity spectra of the type seen in Figure 1 to brightness variations, as observed. To address this, we can use the known amplitude ratio of photometric brightness variability due to heat-driven g-mode oscillations in SPBs to velocity variations measured using spectral line diagnostics in the same stars. The case of convectively driven waves is, of course, different as these waves have shorter lifetimes and some are breaking, leading to turbulence. On the other hand, the heat-driven prograde or retrograde g-modes of SPBs correspond with trapped internal resonant gravity waves. The 2D simulations produce convectively driven resonant gravity waves with amplitudes in the same range as the non-resonant waves. Therefore, in the absence of any better conversion, we assume that the brightness fluctuation signatures due to velocity variations from IGWs behave similarly to those from heat-driven g-modes in terms of amplitude ratios of photometric and spectroscopic observables measured at one instance in time. Under this assumption, we use the carefully measured amplitude ratios of $(\Delta B) /(\Delta\langle\nu\rangle)$ of g-modes to convert tangential velocity spectra to brightness spectra. The measured amplitude ratios for the g-modes in the single SPB pulsators considered in De Cat \& Aerts (2002) have values between roughly 1 and $10 \mathrm{mmag}\left(\mathrm{km} \mathrm{s}^{-1}\right)^{-1}$, as deduced from the light curves in the $B$ band and from the centroid velocity $\langle\nu\rangle$ derived from high-resolution, high signal-to-noise time-series spectroscopy (see Chapter 6 in Aerts et al. 2010 for a definition). Below, we use these observational results to scale wave amplitudes in the simulations for comparison with observations. In the following sections, we first provide observational evidence of the occurrence of IGWs in $\mu$ mag space photometry of three young unevolved O-type stars measured with the CoRoT satellite. We then predict LPVs due to simulated IGWs in order to evaluate if IGWs can provide an alternative explanation of macroturbulent spectral line broadening, in addition to coherent g-mode oscillations (e.g., Aerts et al. 2009; Simón-Díaz et al. 2010). 

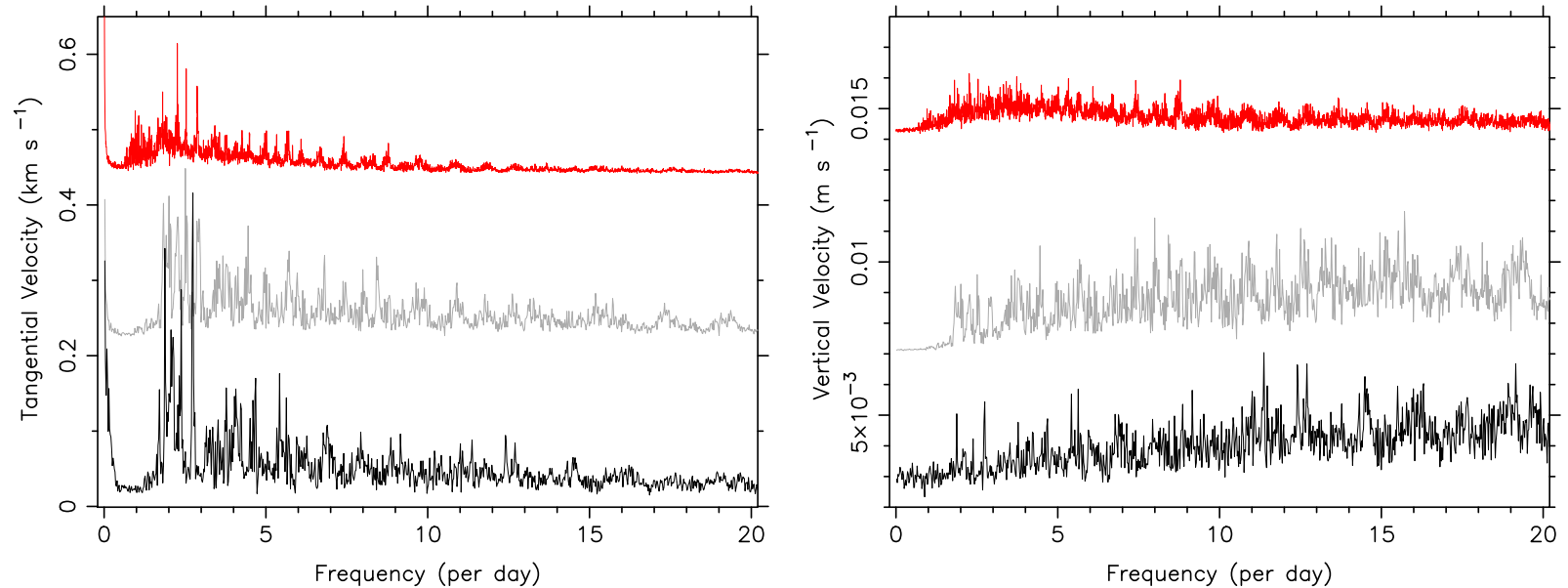

Figure 1. Velocity spectra due to IGWs for three cases: a non-rotating star (black), a rigidly rotating star with initial rotational frequency of 1.1 day ${ }^{-1}$ (gray), and a differentially rotating star with the core rotating 1.5 times faster than the envelope for an initial surface rotational frequency of 0.275 day $^{-1}$ (red), shifted along the $y$-axis for visibility purposes. Left: tangential velocity; right: vertical velocity.

\section{PHOTOMETRIC SIGNATURES: POWER EXCESS IN SPACE PHOTOMETRY OF HOT MASSIVE STARS}

The MOST, CoRoT, and Kepler missions revealed a much larger diversity in variability of OB-type stars at $\mu$ mag level than anticipated prior to the era of high-precision space photometry (e.g., see Neiner et al. 2011; Aerts 2015 for an update). While most of the variability is well understood as being due to heat-driven stellar oscillations, rotational modulation, magnetic activity, mass loss, various sorts of binarity, or a combination of all those processes, the "red noise" power excess found in the amplitude spectra of three $\mathrm{O}$ stars remains unexplained (Blomme et al. 2011).

As is common in the study of heat-driven oscillations, we represent the effect of IGWs by means of amplitude spectra, where we focus on the $\phi$-averaged tangential velocity (see Figure 1). Figure 2 shows the observed amplitude spectra resulting from brightness measurements assembled by the CoRoT mission for three $\mathrm{O}$ stars, with surface rotation frequencies of $0.144 \mathrm{day}^{-1}$ for HD 46150 (top panel), 0.249 day $^{-1}$ for HD 46223 (middle panel), and 0.084 day $^{-1}$ for HD 46966 (bottom panel). These spectra are presented in mmag and have been reproduced from Blomme et al. (2011), who found them to reveal frequencies connected with phenomena of short lifetimes (order of hours to days). We compare those observed spectra (gray line in Figure 2) with the simulated IGW spectra shown in Figure 1 for the non-rigidly rotating case D11 (red line in Figure 2).

We have applied a correction factor of $\sim 0.75$ to the frequencies to account for the factor of $\sim 10$ higher mass between the observed and simulated stars, following Shiode et al. (2013, their Table 1). We realize that the propagation cavity of the IGWs changes somewhat as a function of mass and age, which may imply that such a simple scaling is not optimal. On the other hand, the spectrum of IGWs is dominantly determined by the convective flux and less so by the shape of the Brünt-Väisälä frequency. For this reason, we consider a simple scaling to be the best approach while awaiting future 2D/3D simulations for various stellar masses and evolutionary stages.

We further applied a factor of 2,3 , and 1.5 , respectively, to the IGW amplitude spectra (shown in red in Figure 2) to convert between simulated tangential velocity and brightness, consistent with the observed range of amplitude ratios from
$(\Delta B) /(\Delta\langle\nu\rangle)$ for the heat-driven modes of SPBs. The lowest frequencies, below $1 \mathrm{day}^{-1}$, are not reproduced by the simulations. This could be due to too low a stellar mass and/ or too low a level of differential rotation and/or the too simplified scalings we have adopted. However, the overall resemblance is good in terms of frequency structure. This interpretation of the "red noise" power excess as due to IGWs is currently the only theoretical explanation that provides amplitude spectra whose shape is in agreement with those observed in the three $\mathrm{O}$ stars.

\section{SPECTROSCOPIC SIGNATURES: MACROTURBULENCE IN SPECTRAL LINE PROFILES OF HOT MASSIVE STARS}

High-resolution time-series spectroscopy of confirmed g-mode pulsators shows LPVs of a complex nature, but such data are only available for a handful of SPBs (e.g., De Cat \& Aerts 2002; Pápics et al. 2012). In the absence of systematic time-resolved LPV studies of g-mode pulsators among OBtype stars, Aerts et al. (2009) performed simulations to show that the collective effect of numerous heat-driven g-modes leads to LPVs that mimic the effect of a macroturbulent velocity $\left(v_{\text {macro }}\right)$. More recently, it was shown from observed LPVs that even one mode or spot can give rise to spectral line broadening in B stars consistent with considerable $v_{\text {macro }}$ values (Aerts et al. 2014). These findings indicate that pulsational velocities due to heat-driven g-modes could explain the occurrence of macroturbulence along most of the upper main sequence, except for the highest-mass core-hydrogen-burning $\mathrm{O}$ stars that do not show heat-driven modes (Simón-Díaz 2015). However, they do show a power excess of frequencies with a short lifetime (Figure 2), which we demonstrated may be the signature of IGWs. It is then reasonable to investigate whether or not IGWs can lead to LPVs in these stars.

Using stellar parameters and frequencies of HD 46150 (Blomme et al. 2011; Martins et al. 2015) and velocity fluctuations given in the D11 simulation (scaled by a factor of two as in the red curve in the upper panel of Figure 2), we produced LPVs for its O III $5922 \AA$ line, following the method outlined in Aerts et al. (2010, Chapter 6). In doing so, we used the radial and tangential velocity fluctuations produced in the simulations (Figure 1) as radial and tangential amplitude, 

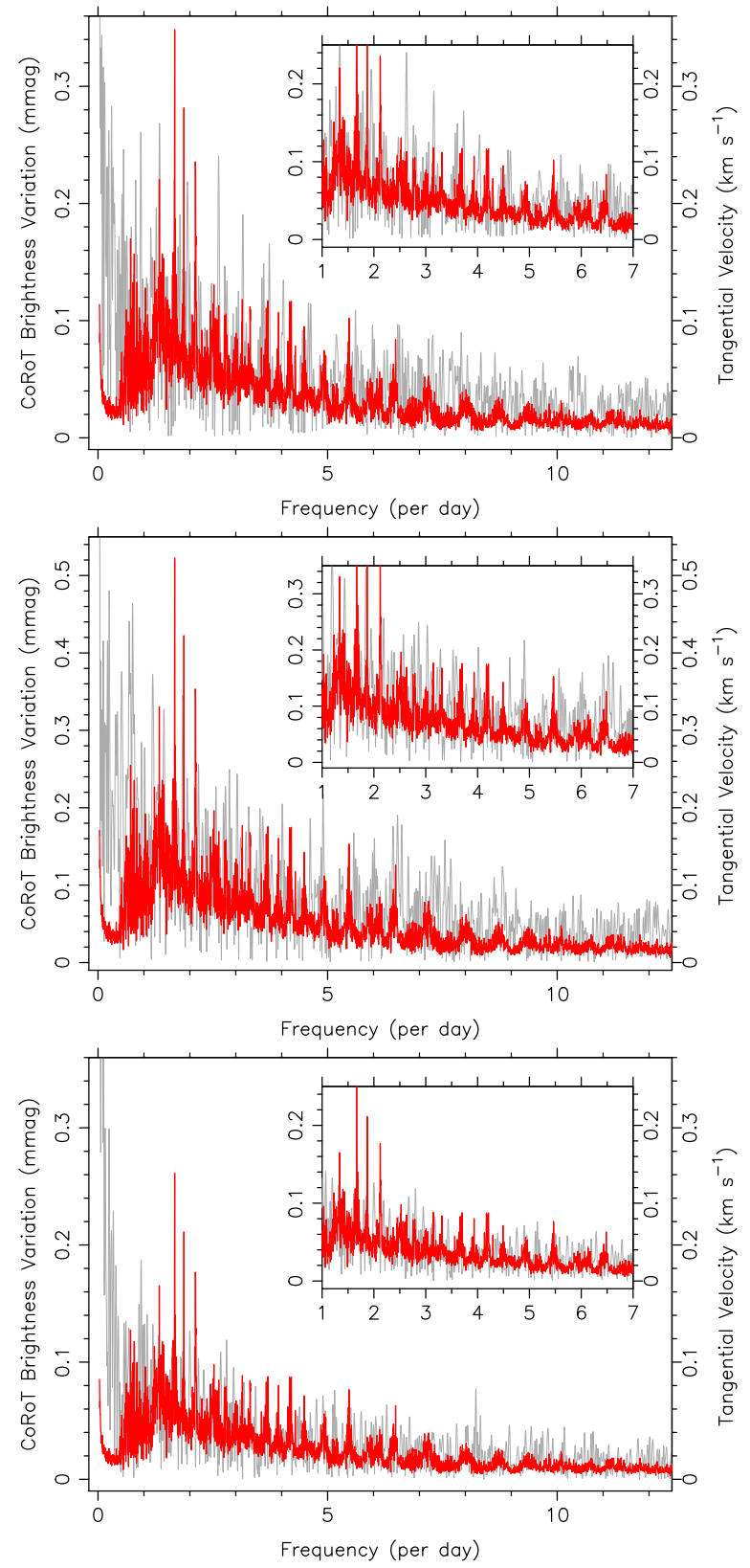

Figure 2. Measured amplitude spectra (gray) overplotted with the predictions of IGWs for case D11 (red) for HD 46150 (top), HD 46223 (middle), and HD 46966 (bottom). The ratios between brightness and tangential velocity variations used are 2,3 , and 1.5 , from top to bottom.

and we adopted a longitudinal and latitudinal dependence according to dipolar prograde spherical harmonics seen under an inclination angle of $60^{\circ}$. This procedure produced the LPVs shown in the left panel of Figure 3. Since there are no observed LPVs for these O-type stars, for comparison, we show simulated LPVs for the SPB star KIC 7760680, representative of its Mg II $4481 \AA$ A line and based upon its identified heat-driven modes detected in the Kepler data. In order to generate these LPVs, we have taken the frequency, amplitude, and phase values from Table 1 in Pápics et al. (2015). The resulting LPVs are shown in the right panel of Figure 3. We find that the velocity fields due to IGWs that give good agreement with the CoRoT photometric data (Figure 2) also lead to LPVs that are quite similar to those produced by heat-driven g-modes of KIC 7760680 and that the spectral line wings of both stars are predicted to be broadened compared to the case of rotational line broadening alone, indicative of macroturbulence. Of course, we have presented just one set of simulated line profiles based on particular assumptions about the nature of IGWs in terms of amplitude and mode type, but these simulations are in agreement with the need for macroturbulence to fit the profiles of HD 46150, as found by Martins et al. (2015; v vacro $\left.\simeq 38 \mathrm{~km} \mathrm{~s}^{-1}\right)$. Our simulations also show that the anticipated LPVs resulting from the chosen angular velocity dependence and inclination angle are tiny in velocity space ( $x$-axis in Figure 3 ) while better visible in relative flux changes ( $y$-axis), i.e., the profiles remain fairly symmetric and fluctuate in relative flux level. So actual future detection of time variability in the O III $5922 \AA$ line of HD 46150 will require very high-resolution, high signal-to-noise time-series spectroscopy in order to observe the fluctuations in the broadening of the spectral line over time, with respect to a timeaveraged profile.

\section{DISCUSSION}

We have shown that the 2D simulations of IGWs by Rogers et al. (2013) give rise to averaged tangential velocity spectra in agreement with measurements of amplitude spectra of flux variations in three mid-O-type stars that do not exhibit heatdriven oscillations. Our simulations of LPVs based on the velocity spectra due to IGWs reveal that such waves can give rise to detectable time-dependent line broadening similar to that observed in SPBs. IGWs thus offer a viable explanation for the occurrence of macroturbulence in the hottest main-sequence stars of spectral type $\mathrm{O}$, which are not subject to heat-driven oscillation modes and/or strictly periodic rotational modulation due to surface spots. Given the shortcomings of the numerical simulations, it is remarkable that they match the observations within factors of two in both frequency and amplitudes. This provides hope that numerical simulations may be able to capture the gross properties of waves in stars and that more sophisticated simulations will be able to make comparisons more robust.

Given our results, it is worthwhile to search for signatures of IGWs in the residual light curves prewhitened for the detected heat-driven mode frequencies for all OB stars observed by the Kepler and CoRoT missions, keeping in mind the recent finding that many of the frequencies found in the amplitude spectra of g-mode pulsators are combination frequencies (possibly due to nonlinear mode coupling) of heat-driven modes (Kurtz et al. 2015; Pápics et al. 2015). However, it should be possible to distinguish between those combination frequencies generated by heat-driven g-modes and the signatures of IGWs since IGWs have short lifetimes leading to frequency features with broad wings in the Fourier transform, while combination frequencies due to heat-driven modes with infinite lifetimes reveal delta-peaks in the frequency spectra. While we provide this interpretation of the nature of macroturbulence in $\mathrm{O}$ stars, an independent spectroscopic study on the nature of macroturbulence has been initiated by Simón-Díaz \& Herrero (2014).

In the near future, we also plan to gather new photometric data with the refurbished Kepler mission, baptized K2 (Howell 2014), accompanied by high-resolution, high-precision spectroscopic time series. Such a combination of data would allow us to test our findings and to provide a large 

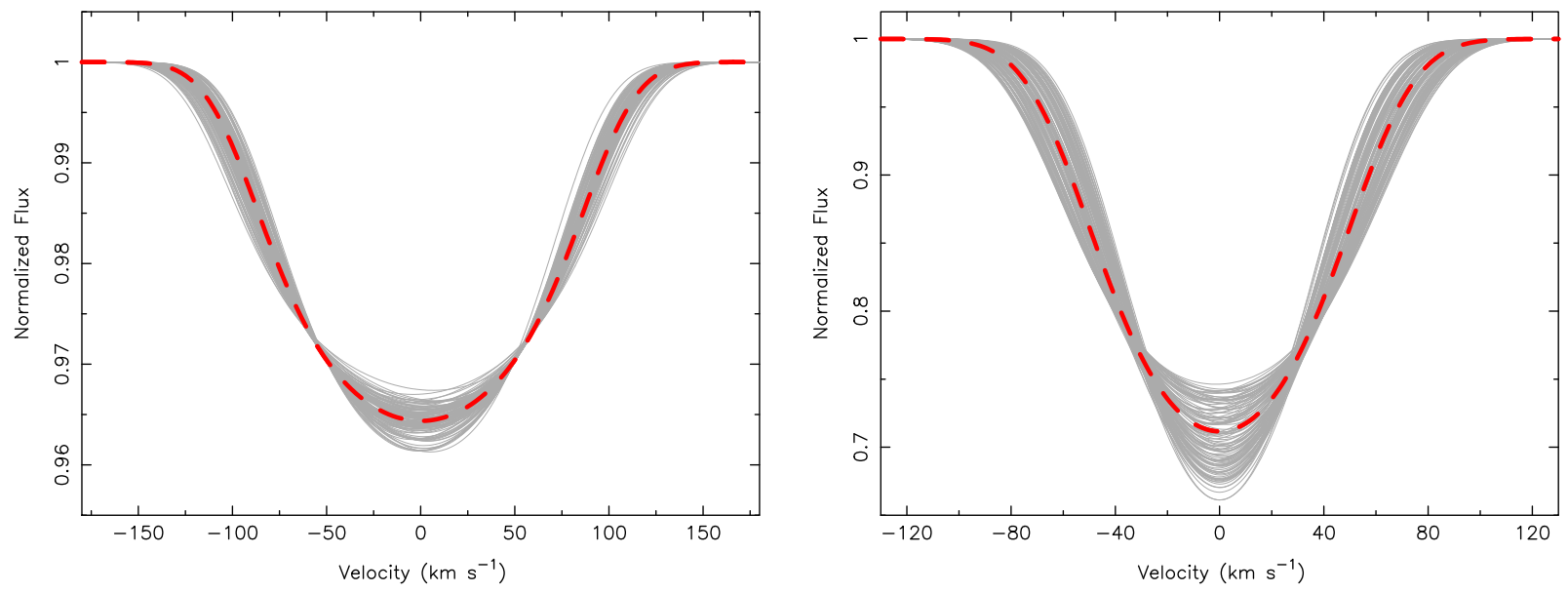

Figure 3. Simulated line-profile variations for the O III $5922 \AA$ line of HD 46150 assuming IGWs (left panel) and for the Mg II $4481 \AA$ A line of the SPB KIC 776068 based on its dipole heat-driven modes detected and identified in the Kepler data (right panel). The red dashed lines are for rotational broadening alone.

enough sample of OB-type stars in all evolutionary phases to test the occurrence of IGWs across the evolution of the most massive stars.

Part of the research included in this Letter was based on funding from the Research Council of KU Leuven, Belgium under grant GOA/2013/012. We thank an anonymous referee for constructive comments that improved this manuscript.

\section{REFERENCES}

Aerts, C. 2015, IAUS, 307, 154

Aerts, C., Christensen-Dalsgaard, J., \& Kurtz, D. W. 2010, Asteroseismology (Heidelberg: Springer)

Aerts, C., Puls, J., Godart, M., \& Dupret, M.-A. 2009, A\&A, 508, 409

Aerts, C., et al. 1999, A\&A, 343, 872

Aerts, C., Simón-Díaz, S., Groot, P. J., \& Degroote, P. 2014, A\&A, 569, A118

Aerts, C., Thoul, A., Daszyńska, J., et al. 2003, Sci, 300, 1926

Ando, H. 1986, A\&A, 163, 97

Belkacem, K., Dupret, M. A., \& Noels, A. 2010, A\&A, 510, A6

Blomme, R., Mahy, L., Catala, C., et al. 2011, A\&A, 533, A4

Browning, M. K., Brun, A. S., \& Toomre, J. 2004, ApJ, 601, 512

De Cat, P., \& Aerts, C. 2002, A\&A, 393, 965

Degroote, P., et al. 2010, Natur, 464, 259
Dziembowski, W. A., Moskalik, P., \& Pamyatnykh, A. A. 1993, MNRAS, 265,588

Gautschy, A., \& Saio, H. 1993, MNRAS, 262, 213

Howell, S. B., et al. 2014, PASP, 126, 398

Kurtz, D. W., Shibahashi, H., Murphy, S. J., Bedding, T. R., \& Bowman, D. M. 2015, MNRAS, in press (arXiv:1504.04245)

Lee, U., Neiner, C., \& Mathis, S. 2014, MNRAS, 443, 1515

Martins, F., et al. 2015, A\&A, 575, A34

Mathis, S., Neiner, C., \& Tran Minh, N. 2014, A\&A, 565, A47

Moravveji, E., Aerts, C., Pápics, P. I., Triana, S. A., \& Vandoren, B. 2015, A\&A, in press (arXiv:1505.06902)

Neiner, C., Floquet, M., Samadi, R., et al. 2012, A\&A, 546, A47

Neiner, C., Wade, G., Meynet, G., \& Peters, G. 2011, IAUS, 272

Pamyatnykh, A. A., Handler, G., \& Dziembowski, W. A. 2004, MNRAS, 350, 1022

Pápics, P. I., Briquet, M., Baglin, A., et al. 2012, A\&A, 542, A55

Pápics, P. I., Moravveji, E., Aerts, C., et al. 2014, A\&A, 570, A8

Pápics, P. I., Tkachenko, A., Aerts, C., et al. 2015, ApJL, 803, L25

Rogers, T. M., Lin, D. N. C., McElwaine, J. N., \& Lau, H. H. B. 2013, ApJ, 772,21

Samadi, R., Belkacem, K., Goupil, M. J., et al. 2010, Ap\&SS, 328, 253

Shiode, J. H., Quataert, E., Cantiello, M., \& Bildsten, L. 2013, MNRAS, 430, 1736

Simón-Díaz, S. 2015, IAUS, 307, 194

Simón-Díaz, S., \& Herrero, A. 2014, A\&A, 562, A135

Simón-Díaz, S., Herrero, A., Uytterhoeven, K., et al. 2010, ApJL, 720, L174

Tassoul, M. 1980, ApJS, 43, 469

Waelkens, C. 1991, A\&A, 246, 453 